in vivo $34: 3473-3481(2020)$

doi:10.21873/invivo.12187

\title{
Predicting Biochemical Failure in Irradiated Patients With Prostate Cancer by Tumour Volume Measured by Multiparametric MRI
}

\author{
BENEDICT OERTHER ${ }^{1}$, MORITZ V. BUREN ${ }^{2}$, CHRISTINA M. KLEIN $^{3,4}$, SIMON KIRSTE $^{3,4}$, \\ NILS H. NICOLAY ${ }^{3,4}$, TANJA SPRAVE ${ }^{3,4}$, SIMON SPOHN ${ }^{3,4}$, DEEPA DARSHINI GUNASHEKAR ${ }^{4,5}$, \\ LEONARD HAGELE ${ }^{4,5}$, LARS BIELAK ${ }^{4,5}$, MICHAEL BOCK ${ }^{4,5}$, ANCA-L. GROSU $^{3,4,6}$, \\ FABIAN BAMBERG ${ }^{1}$, MATTHIAS BENNDORF ${ }^{1}$ and CONSTANTINOS ZAMBOGLOU ${ }^{3,4,6}$ \\ ${ }^{1}$ Department of Radiology, Medical Center - University of Freiburg, \\ Faculty of Medicine, University of Freiburg, Freiburg, Germany; \\ ${ }^{2}$ Department of Urology, Medical Center - University of Freiburg, \\ Faculty of Medicine, University of Freiburg, Freiburg, Germany; \\ ${ }^{3}$ Department of Radiation Oncology, Medical Center - Faculty of Medicine, University of Freiburg, Freiburg, Germany; \\ ${ }^{4}$ German Cancer Consortium (DKTK), Partner Site Freiburg, Freiburg, Germany; \\ ${ }^{5}$ Department of Radiology, Medical Physics, Medical Center - \\ University of Freiburg, Faculty of Medicine, University of Freiburg, Freiburg, Germany; \\ ${ }^{6}$ Berta-Ottenstein-Programme, Faculty of Medicine, University of Freiburg, Freiburg, Germany
}

\begin{abstract}
Background/Aim: We examined the prognostic value of intraprostatic gross tumour volume (GTV) as measured by multiparametric MRI (mpMRI) in patients with prostate cancer following (primary) external beam radiation therapy (EBRT). Patients and Methods: In a retrospective monocentric study, we analysed patients with prostate cancer (PCa) after EBRT. GTV was delineated in pre-treatment mpMRI (GTV-MRI) using T2-weighted images. Coxregression analyses were performed considering biochemical failure recurrence-free survival (BRFS) as outcome variable. Results: Among 131 patients, after a median follow-up of 57 months, biochemical failure occurred in 27 (21\%). GTV-MRI was not correlated with $\%$ of positive biopsy cores, Gleason score and initial PSA (all $r<0.2)$ and only moderately correlated with cT stage $(r=0.32)$. In univariate analysis, $c T$ stage, Gleason score and GTV-MRI were higher in subjects
\end{abstract}

This article is freely accessible online.

Correspondence to: Benedict Oerther, MD, Department of Radiology, Medical Center - University of Freiburg, Faculty of Medicine, University of Freiburg, Hugstetter Straße 55, 79106 Freiburg, Germany. Tel: +49 76127038190, e-mail: benedict.oerther@uniklinik-freiburg.de

Key Words: Prostate cancer, radiation oncology, MRI, tumour volume. with shorter BRFS $(p<0.05)$. GTV-MRI remained a significant predictor for BRFS in multivariate analyses, independent of Gleason score and cT stage. Conclusion: GTV, defined using mpMRI, provides incremental prognostic value for BRFS, independent of established risk factors. This supports the implementation of imaging-based GTV for riskstratification, although further validation is needed.

For patients with primary prostate cancer $(\mathrm{PCa})$, an accurate risk stratification is mandatory to select a suitable treatment. The National Comprehensive Cancer Network riskclassification system for primary PCa patients lists clinical $\mathrm{T}$ stage (cT stage), Gleason score, the percentage of positive biopsy cores (PPBC) and initial prostate-specific antigen (iPSA) levels as important factors (1). Each has been shown to be an independent risk factor of biochemical failure after definitive radiotherapy (RT) (2). However, this model does not always predict the final outcome and its predictions may be very heterogeneous (3). Consequently, more robust riskfactors are urgently needed.

The implementation of multiparametric magnetic resonance imaging (mpMRI) shows promising results in the delineation of the intraprostatic gross tumour volume (GTV) and is considered as the standard of care diagnostic imaging modality for patients with primary $\mathrm{PCa}$ (1). Three studies examined whether local recurrences of PCa after primary RT occur at the location of the primary lesion using pre- and post-treatment MRI in a limited number of patients. Each 
study concluded that local recurrence after RT appears mostly at the primary tumour's location (4-6). Similarly, two studies postulated that even if mpMRI may not detect the entire $\mathrm{PCa}$, it is able to show a clinically sufficient amount of the tumour $(7,8)$. Additionally, a recent study postulated that the RT dose distribution in the MR-defined GTV correlates with outcome after definitive external beam radiotherapy (EBRT) in PCa patients (9).

By calculating the percentage $\operatorname{PPBC}(10,11)$ or by measuring the PCa volume in a surgical specimen $(12,13)$, several studies have proposed that the amount of malignant tissue within the prostate is an independent prognostic factor for patients with PCa. However, it is still not known whether the volume of imaging-defined $\mathrm{PCa}$ is a prognostic factor of the outcome after primary RT. First studies proposed that the intraprostatic tumour extension defined by MRI may be used as a surrogate marker for outcome in patients treated with primary RT $(14,15)$. However, in these studies, only tumour extension in MR images was estimated. In this study, we examined whether the clinically relevant tumour mass can be quantified using GTV that are manually outlined on mpMR images. Consequently, we tested GTV correlation with established prognostic riskfactors and whether GTV may influence the outcome of PCa patients after definitive EBRT.

\section{Patients and Methods}

Patients. In this retrospective, single institution analysis, all patients with a localized and histologically proven PCa who received EBRT with or without androgen deprivation therapy (ADT) were screened from February 2008 to October 2016. The availability of mpMRI data at the maximum of 6 months prior to EBRT was obligatory. Patients were excluded from the analysis in case of $\mathrm{cN} 1$ or $\mathrm{cM} 1$ disease, EBRT of the pelvic lymph nodes, and initial PSA serum values above $50 \mathrm{ng} / \mathrm{ml}$. The administration of ADT over 1 month prior to conduction of MRI scans was also an exclusion criterion. Finally, 131 patients were included in the analysis.

Magnetic resonance imaging. In 116 patients (88.5\%), MR images were acquired on various 1.5 Tesla systems (Siemens Healthcare, Berlin and Munich, Germany), and for 15 patients (11.5\%) a 3 Tesla system (Tim Trio, Siemens Healthcare) was used. For signal reception, a body array surface coil in combination with an integrated spine array coil was used, and no endo-rectal coil was applied. The mpMRI imaging protocol contained a T2-weighted fast spin echo (T2w-TSE) acquisition, diffusion weighted imaging (DWI) and dynamic contrast-enhanced (DCE) perfusion MRI - a detailed list of all acquisition parameters of the mpMRI protocol is given in (16). In case of multiple mpMRI scans before RT, the last pre-treatment scan was selected.

Treatment protocol. EBRT was delivered in $39(30 \%)$ and $92(70 \%)$ patients with 3D-conformal and intensity-modulated RT (IMRT), respectively. All patients had image-guided RT (IGRT) using daily 2D/2D imaging and at least one cone-beam CT per week.
Intraprostatic fiducial markers were implanted in 123 (94\%) patients prior to EBRT. Using the cone-beam CT information, organs at risk as well as target volumes were adapted during IMRT. According to the patient risk stratification the clinical target volume (CTV) was defined. In low-risk patients the prostatic gland and the base of the seminal vesicles were defined as CTV. In intermediate- and highrisk patients the prostatic gland and at least half of the seminal vesicles were expanded by 4-5 $\mathrm{mm}$ (excluding bladder and rectum) to create the CTV. The respective CTVs were expanded with $6 \mathrm{~mm}$ to create the planning target volume (PTV).

The aimed and the delivered median prescription dose to the PTV were 76 and 74 Gy (range=66-78 Gy), respectively. Ten patients had single fractions of $1.8 \mathrm{~Gy}$, and 121 patients had single fractions of 2 Gy. A total of 71 patients (54\%) received ADT parallel to EBRT for a median duration of 7 months (range $=3-24$ months). In the follow-up, patients were seen every 3-6 months for the first two years, and every 6-12 months thereafter for physical examination, PSA measurements and radiological examination, if necessary.

Image co-registration and generation of contours. Axial $\mathrm{T} 2 \mathrm{w}$ sequences and planning CT scans were matched in a RT planning system (Eclipse v13.5, Varian, Palo Alto, CA, USA) using mutual information registration. In case visual assessment showed anatomical mismatch, a manual adjustment was performed. The usage of an axial T2w sequence and at least one DWI- or DCE sequence was obligatory. From the DWI data, the calculated apparent diffusion coefficient (ADC) maps were registered, whereas from the DCE time series a post-injection time frame was manually selected for having a maximum contrast in the prostate. For further alignment between T2w images and the DWI- or DCE sequences the respective pre-set (hardware based) registrations were used.

The prostatic gland (PG) was contoured using CT and T2w-MRI information. The radiology reports of all MRI scans were available during contouring. T2W images were available for all 131 patients, $81(61.8 \%)$ had DWI/ADC images, and for 50 patients (38.2\%) DCE information was additionally available. Considering the PI-RADs v2 criteria (17), a GTV was delineated based on the mpMRI data (GTVMRI) by two readers individually (Figure 1): CZ (GTV-MRI_exp) and BO (GTV-MRI_res). CZ is a board licensed radiation oncologist with over 5 years of experience in prostate MR image interpretation. Additionally, CZ had training according to Rischke et al. (18). BO is a second-year resident at the department of radiology without any additional training in prostate MR image interpretation.

Data analysis and statistics. According to the Phoenix criteria, biochemical failure after EBRT (19) was defined as the study endpoint. The GTV volumes below and above $6 \mathrm{ml}$ were considered as continuous and dichotomous variables - the cut-off point of $6 \mathrm{ml}$ was chosen according to literature $(20,21)$. Univariate and multivariate Cox regression analyses were performed considering biochemical failure recurrence-free survival (BRFS).

For graphical representation, the Kaplan-Meier survival curves for BRFS were used. The Kaplan-Meier curves for the two patient groups (GTV $\leq 6 \mathrm{ml} v s$. GTV $>6 \mathrm{ml}$ ) were compared by a log-rank test. Correlation analyses were performed with nonparametric Spearman rho test. All tests were considered to be statistically significant at $p<0.05$. Statistical analysis was conducted with SPSS v25 (IBM, Armonk, NY, USA). 

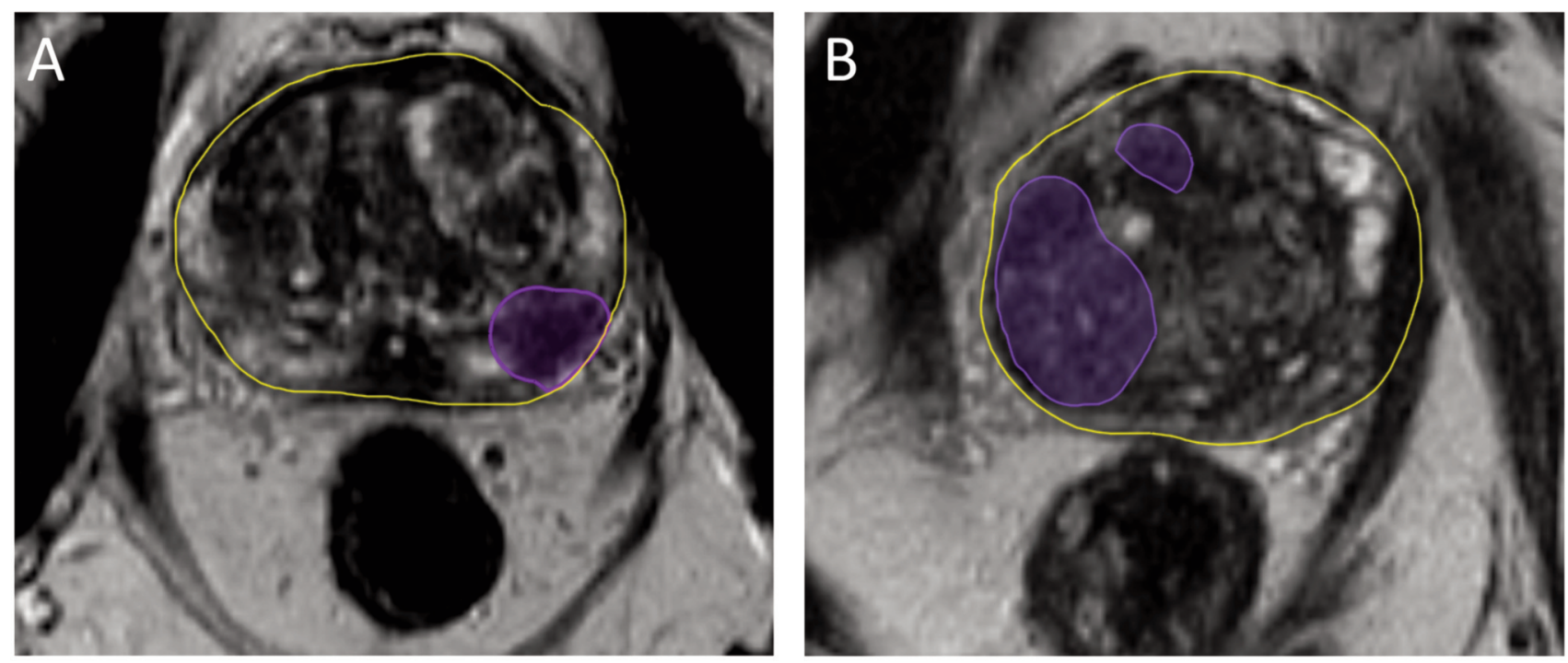

Figure 1. Axial T2-weighted MRI images of patient A and B; purple delineation=gross tumor volume in the current slice. Patient A had tumour stage cT3b cNO cMO, Gleason $7 a(3+4)$ and was 74 years of age at time of diagnosis. He received a radiation dose of 74 Gy and the GTV measured $5 \mathrm{ml}$. Follow-up time was 102 months without biochemical recurrence. Tumour stage of patient B was cT3a cN0 cM0, Gleason 8 . The age was 80 years at time of diagnosis. The mean radiation dose received was $74 \mathrm{~Gy}$. GTV measured $10 \mathrm{ml}$. Biochemical recurrence occurred after $103 \mathrm{months}$.

\section{Results}

Patient and treatment characteristics. Detailed characteristics of the study cohort are listed in Table I. The majority of patients had intermediate-risk disease according to NCNN classification. The median volume of GTVMRI_exp and GTV-MRI_res was $2.6 \mathrm{ml}$ (range $=0-38 \mathrm{ml}$ ) and $2.5 \mathrm{ml}$ (range $=0.2-60.4 \mathrm{ml})$, respectively $(p=0.039 \mathrm{in}$ Wilcoxon matched-pairs test). Among 131 patients (median age $=74$; range $=56-85$ years), after a median follow-up of 57 months (range $=14-138$ months) following EBRT, biochemical failure occurred in $27(21 \%)$. Median PSA level at the time of recurrence was $4.5 \mathrm{ng} / \mathrm{ml}$ (range $=2.3-14.9$ $\mathrm{ng} / \mathrm{ml}$ ). At the time of last evaluation 2 patients died of PCa. Univariate Cox-regression showed that the prescription dose, the RT technique (IMRT vs. 3D) and the usage of ADT had no impact on BRFS $(p>0.05)$.

Correlation between GTV-MRI and established prognostic factors for BRFS. For the GTV contours of both readers a very weak correlation was observed with PPBC, Gleason score and initial PSA (Spearman $\mathrm{r}<20, p>0.05$ ). A weak correlation with the cT stage was found for GTV-MRI_exp (Spearman $\mathrm{r}=0.31, p<0.01$ ) and GTV-MRI_res (Spearman $\mathrm{r}=0.33, p<0.01$ ), respectively. The correlation between GTVMRI_exp volumes and clinical parameters is presented in Figure 2 .

Impact of clinical parameters on BRFS. The Kaplan-Meier curves (Figure 3) demonstrate the impact of absolute GTV-
Table I. Patient characteristics.

\begin{tabular}{lc}
\hline & Total $(\mathrm{n}=131)$ \\
\hline Median age in years (range) & $74(56-85)$ \\
Median initial PSA in ng/ml (range) & $10.1(2-49.2)$ \\
Biopsy Gleason score, $\mathrm{n}(\%)$ & \\
6 & $19(15)$ \\
$7 \mathrm{a}$ & $47(36)$ \\
$7 \mathrm{~b}$ & $39(30)$ \\
8 & $16(12)$ \\
9 & $10(8)$ \\
10 & 0 \\
Median PPBC (range) & $39(5-100)$ \\
Clinical T stage, $\mathrm{n}(\%)$ & \\
2a & $20(16)$ \\
2b & $22(17)$ \\
2c & $51(38)$ \\
3a & $29(22)$ \\
3b & $9(7)$ \\
NCNN risk group, $\mathrm{n}(\%)$ & \\
Low-risk & $2(1)$ \\
Intermediate-risk & $71(54)$ \\
High-risk & $58(44)$ \\
Median Volume, ml (range) & \\
PG & 36.9 \\
GTV-MRI_exp & $2.6(0-38)$ \\
GTV-MRI_res & $2.5(0.2-60.4)$ \\
Median follow-up time, months (range) & $57(14-138)$ \\
BR, n (\%) & $27(21)$ \\
\hline
\end{tabular}

NCCN risk-groups: Very low and low-risk patients are subsummarized as low-risk patients and very high and high-risk patients are sub summarized as high-risk patients. n: Number of patients; PG: prostatic gland; GTV: intraprostatic gross tumour volume; PPBC: \% of positive biopsy cores; BR: biochemical recurrences. 

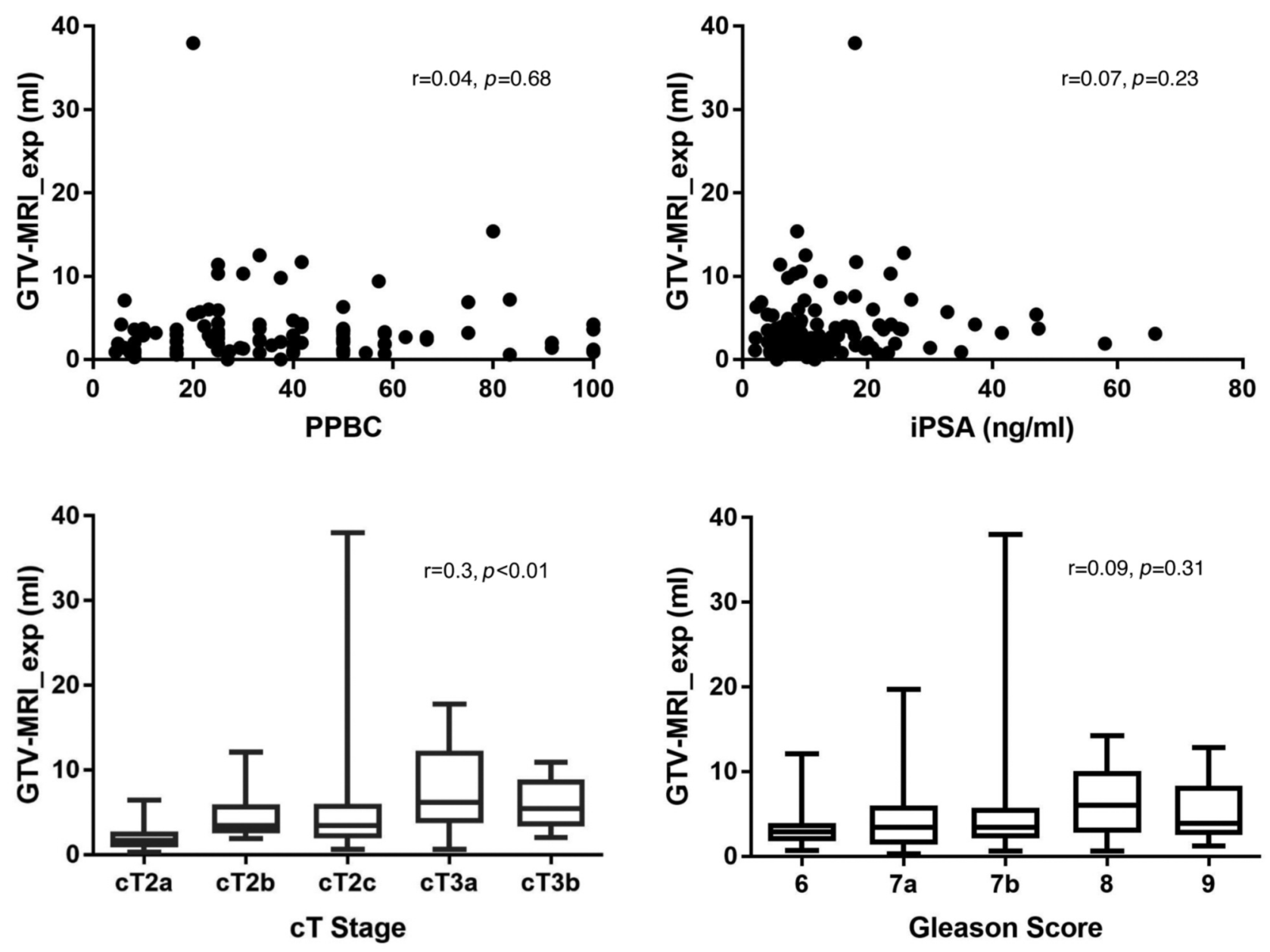

Figure 2. Correlation analysis of absolute volume of GTV-MRI_exp with cT stage, Gleason score, initial PSA and PPBC. For each cT stage or Gleascon score the respective box plots are presented (including median, minimum and maximum values) and Spearman $r$ values with the respective p-values listed.

MRI volumes (cut-off: $6 \mathrm{ml}$ ) on BRFS. Table II shows the results of Cox regression analyses to assess the impact of different patient-related factors on BRFS. Univariate analyses revealed that 3 out of 10 factors were statistically significant $(p<0.05)$ : volume of GTV-MRI, Gleason score and cT stage. In multivariate analysis, including all significant parameters from the univariate analysis, the volume of GTV-MRI_exp (cut-off: $6 \mathrm{ml}$ and continuous variable), the volume of GTV-MRI_res (cut-off: $6 \mathrm{ml}$ ) and the Gleason score were independent risk factors for BRFS.

\section{Discussion}

The NCCN risk-stratification system is widely used for patients with primary $\mathrm{PCa}$, although it may not always predict the outcome correctly (3). To improve riskstratification systems, several studies showed that the PCa amount has an impact on the outcome of patients treated with prostatectomy or EBRT for primary PCa $(10-12,22)$. However, in most patients treated with primary RT, the size of the PCa was indirectly assessed by PPBC (23) or by disease localization based on MRI imaging information (2426). Kaufmann et al. reported that the largest axial tumour diameter in MR imaging was strongly associated with biochemical outcomes after RT for primary PCa (26). In this study, we postulated that mpMRI may define the significant PCa amount. Likewise, we hypothesized that the volume of the manually outlined GTV-MRI should predict the outcome in terms of biochemical failure after primary EBRT.

With multivariate Cox regression analyses, we demonstrated that a tumour volume of more than $6 \mathrm{ml}$ is an independent prognostic factor for BRFS, suggesting an up to 3-fold increase in the likelihood of biochemical tumour recurrence. Additionally, when defined by an expert, the absolute volume can be used as a continuous variable where each 1 ml-increase of GTV volume leads to an about $10 \%$ 


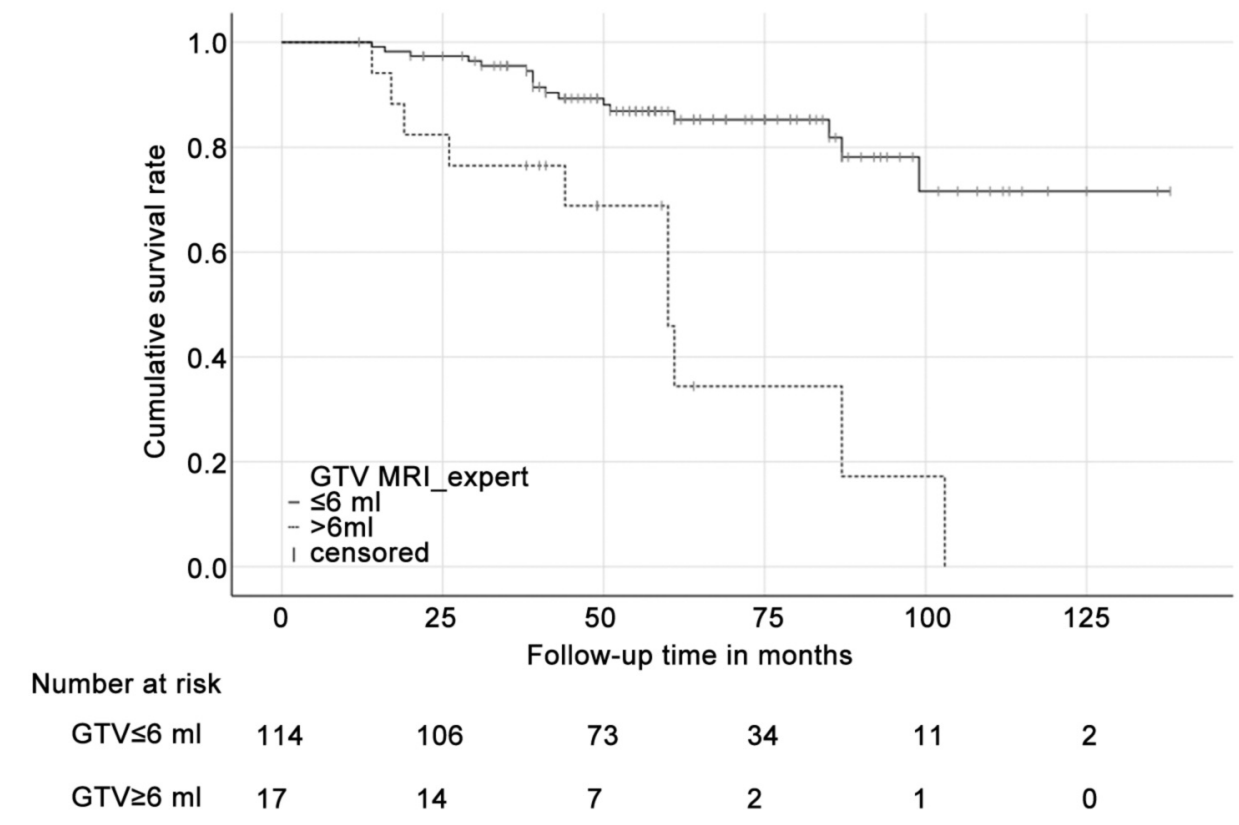

Figure 3. Kaplan-Meier curve for BRFS. Statistical comparison with Log-rank test revealed a $p=0.001$ on absolute volume of GTV-MRI_exp (cutoff value: $6 \mathrm{ml}$ ).

increase in BRFS risk. These findings may be explained by the increased number of clonogenic PCa cells (27) or by a possible increase in the number of hypoxic regions in larger tumours (28). Furthermore, McNeal et al. postulated that the $\mathrm{PCa}$ volume in surgery specimens strongly correlates with nodal metastases (29). Kim et al. (12) used a threshold of $5 \mathrm{ml}$ to categorize extensive $\mathrm{PCa}$ in prostatectomy specimens, which is close to our $6 \mathrm{ml}$-threshold. The cutoff value of $6 \mathrm{ml}$ was also suggested as a predictive factor for worse outcomes by the prostatectomy studies of Stamney et al. $(20,21)$.

Several previous studies had a huge interobserver variability for manually delineated GTVs based on MR images $(18,30)$. In this study, two observers with different levels of experience were contouring the GTVs. No statistically significant differences in absolute GTV-volumes were observed, and the cut-off value of $6 \mathrm{ml}$ was a significant predictor of BRFS for both readers. However, only the expert contour had a significant impact on BRFS as a continuous variable, indicating that the variations in the contours drawn by the novice may lead to fluctuations that mask the correlation between GTV volume and outcome. Future studies should examine whether implementation of automatic GTV segmentation methods based on deep convolutional neuronal networks may improve the robustness of GTV segmentation (31).

In our study, no correlation was found between iPSA serum values and GTV-MRI, which may be explained by the non-specific PSA expression of non-malignant cells in the prostate and by other non-tumour specific factors that can influence PSA expression (32). Interestingly, only a weak correlation between cT stage and GTV-MRI was observed: patients with cT3b disease had lower median GTV-MRI values compared to cT3a PCa patients.

GTV-MRI did not correlate with Gleason score, which is consistent with Donato et al. who did not find a correlation between PCa volume in ADC-MRI and Gleason score (33). Furthermore, no correlation between PPBC and GTV-MRI was detected, and some of the patients with PPBC $>80 \%$ had low GTV-MRI volumes $(<2 \mathrm{ml})$. It should be mentioned that an exact reconstruction of the intraprostatic tumour mass is not assured in trans-rectal biopsies (34), which may explain the missing correlation between PPBC and GTV volume. Only $4 \%$ of the patients received a transperineal TRUSfusion biopsy (guided by mpMRI or PSMA PET/CT). Future studies should analyse whether the volume of GTV correlates better with PPBC and Gleason score after transperineal TRUS-fusion biopsies. Additionally, the usage of ADT between biopsy and pre-RT images may serve as an explanation for the lack of correlation between PPBC and GTV-MRI. ADT administration might shrink the volume of the lesion (35), moving some patients under the threshold even if the original volume of the GTV was $\geq 6 \mathrm{~mm}$. In our study, 25 of the 71 patients $(35 \%)$ with ADT received mpMRI scans during ADT. Future studies are needed to evaluate whether GTV for risk-stratification should be derived before or after ADT. In summary, our findings suggest that GTV-MRI volume may offer complementary 
Table II. Cox-regression analyses considering BRFS after external beam radiotherapy.

\begin{tabular}{lc}
\hline & Univariate analysis \\
\cline { 2 - 2 } Patient parameter & $p$-Value \\
\hline iPSA in ng/ml $(<10,10-20,>20)$ & 0.226 \\
Gleason score in biopsy $(<7,7,>7)$ & $\mathbf{0 . 0 0 1}$ \\
cT stage $(2$ vs. 3) & $\mathbf{0 . 0 2 2}$ \\
Volume GTV-MRI_exp in $\mathrm{ml}(\leq 6,>6)$ & $\mathbf{0 . 0 0 1}$ \\
Volume GTV-MRI_res in $\mathrm{ml}(\leq 6,>6)$ & $\mathbf{0 . 0 0 1}$ \\
Volume GTV-MRI_exp in ml (continuous) & $\mathbf{0 . 0 0 5}$ \\
Volume GTV-MRI_res in ml (continuous) & 0.059 \\
PSA density in ng/ml (continuous) & 0.994 \\
PSA tumour density in ng/ml (continuous) & 0.693 \\
Age in years (continuous) & 0.331 \\
PPBC $(<50, \geq 50)(13)$ & 0.794 \\
\hline
\end{tabular}

\begin{tabular}{lcr}
\hline & \multicolumn{2}{c}{ Multivariate analysis } \\
\cline { 2 - 3 } & HR $(95 \% \mathrm{CI})$ & $p$-Value \\
\hline Gleason score in biopsy $(<7,7,>7)$ & $\mathbf{2 . 3}(\mathbf{1 . 2}-\mathbf{4 . 6})$ & $\mathbf{0 . 0 1 4}$ \\
cT stage $(2$ vs. 3) & $1.7(0.8-3.7)$ & 0.206 \\
Volume GTV-MRI_exp in ml $(\leq 6,>6)$ & $\mathbf{3 . 4}(\mathbf{1 . 5}-\mathbf{7 . 9})$ & $\mathbf{0 . 0 0 4}$ \\
\hline Gleason score in biopsy $(<7,7,>7)$ & $\mathbf{2 . 7}(\mathbf{1 . 4 - 5 . 3})$ & $\mathbf{0 . 0 0 4}$ \\
cT stage $(2$ vs. 3) & $1.8(0.8-3.8)$ & 0.146 \\
Volume GTV-MRI_exp in ml $($ continuous $)$ & $1.1(1-1.1)$ & $\mathbf{0 . 0 4 1}$ \\
\hline Gleason score in biopsy $(<7,7,>7)$ & $2.4(1.2-4.7)$ & 0.015 \\
cT stage $(2$ vs. 3) & $1.8(0.8-3.9)$ & 0.161 \\
Volume GTV-MRI_res in ml $(\leq 6,>6)$ & $\mathbf{2 . 7}(\mathbf{1 . 2}-\mathbf{6 . 2})$ & $\mathbf{0 . 0 2 0}$ \\
\hline
\end{tabular}

Results of the Cox regression analysis for the influence of different patient-related parameters on BRFS. Cut-off value for PPBC was derived from the respective reference. Multivariate analyses were adjusted for Gleason score and for cT stage and performed for continuous and dichotomous GTV-MRI volumes. PSA-density: PSA level in $\mathrm{ng} / \mathrm{ml}$ in relation to volume of the prostate in $\mathrm{ml}$; PSA-tumourdensity: PSA level in $\mathrm{ng} / \mathrm{ml}$ in relation to GTV in $\mathrm{ml}$. CI: Confidence interval; HR: hazard ratio. Bold: values with $p<0.05$.

information to the NCCN risk-stratification system as an independent risk factor. It would be interesting to further investigate the correlation of other parameters extracted from GTV-MRI and the corresponding prognosis. Two studies proposed the usage of diffusion-weighted MRI as an imaging biomarker for monitoring response of $\mathrm{PCa}$ to primary $\mathrm{RT}$ $(36,37)$. Turchan et al. showed that PCa lesions with PIRADs score of 5 increase the risk of failure after RT for primary $\mathrm{PCa}(38)$.

This study has several limitations due to its retrospective character: treatment protocols (e.g. ADT duration, RT technique) were not identical for all patients. However, RT technique, RT dose and the usage of ADT had no significant impact on BRFS in our study. Furthermore, MRI systems and acquisition protocols were not identical for the entire group, but imaging contrasts such as T2, diffusion coefficient and perfusion-induced contrast change were used that are robust against different field strengths and imaging hardware. Despite the usage of different imaging methods and imaging protocols, we showed that image-defined GTV had a significant impact on BRFS. Interestingly, we reproduced the cut-off value of 6 $\mathrm{ml}$ for GTV, which was already described by prostatectomy studies. Additionally, the usage of different MRI systems and protocols reflects the current clinical situation, and every new generalizable biomarker for risk prediction has to be robust in various clinical settings: a mandatory condition for the implementation of imaging-derived GTV for risk stratification systems must be a maximum independence of the obtained results from the utilised imaging system. Another limitation of our study is the relatively short follow-up time (approx. 5 years), which did not enable the evaluation of other relevant endpoints like PCa-specific survival or overall survival. However, other similar studies had even shorter follow-up times of 43-44 months $(24,39)$. Although mpMRI imaging is the standard of care for primary PCa staging, a higher sensitivity for PSMA PET/CT in GTV detection was reported $(40,41)$. Future studies should address whether PSMA-based GTV may also be used for risk prediction of patients with primary PCa. To account for these issues, our findings need external validation, preferably from prospective studies with longer follow-up.

This study showed that GTV defined on mpMRI has a weak correlation with known prognostic factors for risk stratification in patients with primary PCa. GTV derived from pre-therapeutic MR images is an independent predictive factor for biochemical failure after EBRT. These findings support the implementation of GTV delineation based on modern imaging tools for risk-stratification systems in patients with primary $\mathrm{PCa}$.

\section{Conflicts of Interest}

All Authors disclose that no financial and personal relationships with other people or organisations that could inappropriately influence the work exist.

\section{Authors' Contributions}

BO acquired clinical follow-up data, delineated GTV in mpMRI images, carried out statistical analysis and was a major contributor to the final manuscript. MVB acquired primary, clinical follow-up data and patients. CK acquired primary and clinical follow-up data, delineated GTV in mpMRI images. SK acquired primary and clinical follow-up data. NN acquired primary and clinical followup data and was a major contributor to the final manuscript. TS acquired primary and clinical follow-up data. SS acquired primary and clinical follow-up data. DDG carried out statistical analysis. LH extracted imaging information and processed delineated GTV. LB extracted imaging information and processed delineated GTV. MBo 
carried out statistical analysis and was a major contributor to the final manuscript. AG acquired primary and clinical follow-up data and was a major contributor to the final manuscript. FB supervised imaging acquisition and was a major contributor to the final manuscript. MBe supervised imaging acquisition and was a major contributor to the final manuscript. $\mathrm{CZ}$ supervised imaging acquisition acquired primary and follow-up clinical data, delineated GTV in mpMRI images, carried out statistical analysis and was a major contributor to the final manuscript.

\section{References}

1 Mohler JL and Antonarakis ES: NCCN guidelines updates: Management of prostate cancer. J Natl Compr Canc Netw 17(5.5): 583-586, 2019. PMID: 31117038. DOI: 10.6004/jncen. 2019.5011

2 D'Amico AV, Whittington R, Malkowicz SB, Schultz D, Blank K, Broderick GA, Tomaszewski JE, Renshaw AA, Kaplan I, Beard CJ and Wein A: Biochemical outcome after radical prostatectomy, external beam radiation therapy, or interstitial radiation therapy for clinically localized prostate cancer. JAMA 280(11): 969-974, 1998. PMID: 9749478. DOI: 10.1001/jama. 280.11.969

3 Williams SG, Duchesne GM, Gogna NK, Millar JL, Pickles T, Pratt GR and Turner S: An international multicenter study evaluating the impact of an alternative biochemical failure definition on the judgment of prostate cancer risk. Int J Radiat Oncol Biol Phys 65(2): 351-357, 2006. PMID: 16530339. DOI: 10.1016/j.ijrobp.2005.12.007

4 Arrayeh E, Westphalen AC, Kurhanewicz J, Roach M, Jung AJ, Carroll PR and Coakley FV: Does local recurrence of prostate cancer after radiation therapy occur at the site of primary tumor? Results of a longitudinal mri and mrsi study. Int J Radiat Oncol Biol Phys 82(5): E787-E793, 2012. PMID: 22331003. DOI: 10.1016/j.ijrobp.2011.11.030

5 Pucar D, Hricak H, Shukla-Dave A, Kuroiwa K, Drobnjak M, Eastham J, Scardino PT and Zelefsky MJ: Clinically significant prostate cancer local recurrence after radiation therapy occurs at the site of primary tumor: Magnetic resonance imaging and stepsection pathology evidence. Int J Radiat Oncol Biol Phys 69(1): 62-69, 2007. PMID: 17707266. DOI: 10.1016/j.ijrobp.2007.03.065

6 Mendez LC, Ravi A, Chung H, Tseng CL, Wronski M, Paudel M, McGuffin M, Cheung P, Loblaw A and Morton G: Pattern of relapse and dose received by the recurrent intraprostatic nodule in low- to intermediate-risk prostate cancer treated with single fraction 19 gy high dose-rate brachytherapy. Brachytherapy, 2017. PMID: 29137956. DOI: 10.1016/j.brachy.2017.10.001

7 Cash H, Maxeiner A, Stephan C, Fischer T, Durmus T, Holzmann J, Asbach P, Haas M, Hinz S, Neymeyer J, Miller K, Gunzel K and Kempkensteffen C: The detection of significant prostate cancer is correlated with the prostate imaging reporting and data system (pi-rads) in mri/transrectal ultrasound fusion biopsy. World J Urol 34(4): 525-532, 2016. PMID: 26293117. DOI: $10.1007 / \mathrm{s} 00345-015-1671-8$

8 Yuan Q, Costa DN, Senegas J, Xi Y, Wiethoff AJ, Rofsky NM, Roehrborn C, Lenkinski RE and Pedrosa I: Quantitative diffusion-weighted imaging and dynamic contrast-enhanced characterization of the index lesion with multiparametric mri in prostate cancer patients. J Magnetic Resonance Imaging 45(3): 908-916, 2017. PMID: 27442039. DOI: 10.1002/jmri.25391
9 Zamboglou C, Klein CM, Thomann B, Fassbender TF, Rischke HC, Kirste S, Henne K, Volegova-Neher N, Bock M, Langer M, Meyer PT, Baltas D and Grosu AL: The dose distribution in dominant intraprostatic tumour lesions defined by multiparametric mri and psma pet/ct correlates with the outcome in patients treated with primary radiation therapy for prostate cancer. Radiat Oncol 13(1): 65, 2018. PMID: 5898009. DOI: 10.1186/s 13014-018-1014-1

10 D'Amico AC, Renshaw AA, Cote K, Hurwitz M, Beard C, Loffredo $\mathrm{M}$ and Chen MH: Impact of the percentage of positive prostate cores on prostate cancer-specific mortality for patients with low or favorable intermediate-risk disease. J Clin Oncol 22(18): 3726-3732, 2004. PMID: 15365069. DOI: 10.1200/ Jco.2004.01.164

11 Huang J, Vicini FA, Williams SG, Ye H, McGrath S, Ghilezan M, Krauss D, Martinez AA and Kestin LL: Percentage of positive biopsy cores: A better risk stratification model for prostate cancer? Int J Radiat Oncol Biol Phys 83(4): 1141-1148, 2012. PMID: 22099043. DOI: 10.1016/j.ijrobp.2011.09.043

12 Kim KH, Lim SK, Shin TY, Kang DR, Han WK, Chung BH, Rha $\mathrm{KH}$ and Hong SJ: Tumor volume adds prognostic value in patients with organ-confined prostate cancer. Ann Surg Oncol 20(9): 3133-3139, 2013. PMID: 23720069. DOI: 10.1245/ s10434-013-3016-4

13 Ghadjar P, Hayoz S, Genitsch V, Zwahlen DR, Holscher T, Gut P, Guckenberger M, Hildebrandt G, Muller AC, Putora PM, Papachristofilou A, Stalder L, Biaggi-Rudolf C, Sumila M, Kranzbuhler H, Najafi Y, Ost P, Azinwi NC, Reuter C, Bodis S, Khanfir K, Budach V, Aebersold DM, Thalmann GN and the Swiss Group for Clinical Cancer Research (SAKK): Importance and outcome relevance of central pathology review in prostatectomy specimens: Data from the sakk 09/10 randomized trial on prostate cancer. BJU Int 120(5B): E45-E51, 2017. PMID: 27987524. DOI: 10.1111/bju.13742

14 Riaz N, Afaq A, Akin O, Pei X, Kollmeier MA, Cox B, Hricak $\mathrm{H}$ and Zelefsky MJ: Pretreatment endorectal coil magnetic resonance imaging findings predict biochemical tumor control in prostate cancer patients treated with combination brachytherapy and external-beam radiotherapy. Int J Radiat Oncol Biol Phys 84(3): 707-711, 2012. PMID: 22425220. DOI: 10.1016/j.ijrobp.2012.01.009

15 Fuchsjager MH, Pucar D, Zelefsky MJ, Zhang ZG, Mo QX, Ben-Porat LS, Shukla-Dave A, Wang LA, Reuter VE and Hricak $\mathrm{H}$ : Predicting post-external beam radiation therapy psa relapse of prostate cancer using pretreatment mri. Int J Radiat Oncol Biol Phys 78(3): 743-750, 2010. PMID: 20133067. DOI: 10.1016/j.ijrobp.2009.08.040

16 Zamboglou C, Wieser G, Hennies S, Rempel I, Kirste S, Soschynski M, Rischke HC, Fechter T, Jilg CA, Langer M, Meyer PT, Bock M and Grosu AL: Mri versus (68)ga-psma pet/ct for gross tumour volume delineation in radiation treatment planning of primary prostate cancer. Eur J Nucl Med Mol Imaging 43(5): 889-897, 2016. PMID: 26592938. DOI: $10.1007 / \mathrm{s} 00259-015-3257-5$

17 Turkbey B, Rosenkrantz AB, Haider MA, Padhani AR, Villeirs G, Macura KJ, Tempany CM, Choyke PL, Cornud F, Margolis DJ, Thoeny HC, Verma S, Barentsz J and Weinreb JC: Prostate imaging reporting and data system version 2.1: 2019 update of prostate imaging reporting and data system version 2. Eur Urol 76(3): 340351, 2019. PMID: 30898406. DOI: 10.1016/j.eururo.2019.02.033 
18 Rischke HC, Nestle U, Fechter T, Doll C, Volegova-Neher N, Henne K, Scholber J, Knippen S, Kirste S, Grosu AL and Jilg CA: 3 tesla multiparametric mri for gtv-definition of dominant intraprostatic lesions in patients with prostate cancer-an interobserver variability study. Radiat Oncol 8: 183, 2013. PMID: 3828667. DOI: 10.1186/1748-717X-8-183

19 Roach M, Hanks G, Thames H, Schellhammer P, Shipley WU, Sokol GH and Sandler H: Defining biochemical failure following radiotherapy with or without hormonal therapy in men with clinically localized prostate cancer: Recommendations of the rtog-astro phoenix consensus conference. Int J Radiat Oncol Biol Phys 65(4): 965-974, 2006. PMID: 16798415. DOI: 10.1016/j.ijrobp.2006.04.029

20 Stamey TA, McNeal JE, Yemoto CM, Sigal BM and Johnstone IM: Biological determinants of cancer progression in men with prostate cancer. JAMA 281(15): 1395-1400, 1999. PMID: 10217055. DOI: 10.1001/jama.281.15.1395

21 Noguchi M, Stamey TA, McNeal JE and Yemoto CM: Preoperative serum prostate specific antigen does not reflect biochemical failure rates after radical prostatectomy in men with large volume cancers. J Urol 164(5): 1596-1600, 2000. PMID: 11025712. DOI: 10.1016/S0022-5347(05)67036-X

22 Freedland SJ, Aronson WJ, Terris MK, Kane CJ, Amling CL, Dorey F, Presti JC and Grp SDS: Percent of prostate needle biopsy cores with cancer is a significant independent predictor of prostate specific antigen recurrence following radical prostatectomy: Results from the search database. J Urol 169(6): 2136-2141, 2003. PMID: 12771735. DOI: 10.1097/01.ju.0000065588.82511.06

23 D'Amico AV, Renshaw AA, Cote K, Hurwitz M, Beard C, Loffredo $\mathrm{M}$ and Chen $\mathrm{MH}$ : Impact of the percentage of positive prostate cores on prostate cancer-specific mortality for patients with low or favorable intermediate-risk disease. J Clin Oncol 22(18): 3726-3732, 2004. PMID: 15365069. DOI: 10.1200/ JCO.2004.01.164

24 McKenna DA, Coakley FV, Westphalen AC, Zhao S, Lu Y, Webb EM, Pickett B, Roach M, 3rd and Kurhanewicz J: Prostate cancer: Role of pretreatment $\mathrm{mr}$ in predicting outcome after external-beam radiation therapy-initial experience. Radiology 247(1): 141-146, 2008. PMID: 3694427. DOI: 10.1148/radiol. 2471061982

25 Fuchsjager MH, Pucar D, Zelefsky MJ, Zhang Z, Mo Q, BenPorat LS, Shukla-Dave A, Wang L, Reuter VE and Hricak H: Predicting post-external beam radiation therapy psa relapse of prostate cancer using pretreatment mri. Int J Radiat Oncol Biol Phys 78(3): 743-750, 2010. PMID: 2891893. DOI: 10.1016/ j.ijrobp.2009.08.040

26 Kauffmann G, Arif F, Patel P, Oto A and Liauw SL: Pretreatment multiparametric mri is independently associated with biochemical outcome in men treated with radiation therapy for prostate cancer. Urol Oncol 36(10), 2018. PMID: 30122344. DOI: 10.1016/j.urolonc.2018.07.004

27 Bentzen SM and Thames HD: Tumor volume and local control probability: Clinical data and radiobiological interpretations. Int J Radiat Oncol Biol Phys 36(1): 247-251, 1996. PMID: 8823282. DOI: $10.1016 / \mathrm{s} 0360-3016(96) 00242-8$

28 Dunst J, Stadler P, Becker A, Lautenschlager C, Pelz T, Hansgen G, Molls M and Kuhnt T: Tumor volume and tumor hypoxia in head and neck cancers - the amount of the hypoxic volume is important. Strahlenther Onkol 179(8): 521-526, 2003. PMID: 14509950. DOI: 10.1007/s00066-003-1066-4
29 McNeal JE, Villers AA, Redwine EA, Freiha FS and Stamey TA: Histologic differentiation, cancer volume, and pelvic lymph node metastasis in adenocarcinoma of the prostate. Cancer 66(6): 1225-1233, 1990. PMID: 2400973. DOI: 10.1002/10970142(19900915)66:6<1225::aid-cncr2820660624>3.0.co;2-x

30 Steenbergen P, Haustermans K, Lerut E, Oyen R, De Weyer L, Van den Bergh L, Kerkmeijer LGW, Pameijer FA, Veldhuis WB, van Zyp JRNV, Pos FJ, Heijmink SW, Kalisvaart R, Teertstra HJ, Dinh CV, Ghobadi G and van der Heide UA: Prostate tumor delineation using multiparametric magnetic resonance imaging: Inter-observer variability and pathology validation. Radiother Oncol 115(2): 186-190, 2015. PMID: 25935742. DOI: 10.1016/ j.radonc 2015.04 .012

31 Bielak L, Wiedenmann N, Nicolay NH, Lottner T, Fischer J, Bunea H, Grosu AL and Bock M: Automatic tumor segmentation with a convolutional neural network in multiparametric mri: Influence of distortion correction. Tomography 5(3): 292-299, 2019. PMID: 31572790 . DOI: 10.18383/j.tom.2019.00010

32 Beebe-Dimmer JL, Faerber GJ, Morgenstern H, Werny D, Wojno K, Halstead-Nussloch B and Cooney KA: Body composition and serum prostate-specific antigen: Review and findings from flint men's health study. Urology 71(4): 554-560, 2008. PMID: 18308373. DOI: 10.1016/j.urology.2007.11.049

33 Donati OF, Afaq A, Vargas HA, Mazaheri Y, Zheng JT, Moskowitz CS, Hricak H and Akin O: Prostate mri: Evaluating tumor volume and apparent diffusion coefficient as surrogate biomarkers for predicting tumor gleason score. Clin Cancer Res 20(14): 3705-3711, 2014. PMID: 24850842. DOI: 10.1158/10780432.CCR-14-0044

34 Eichler K, Hempel S, Wilby J, Myers L, Bachmann LM and Kleijnen J: Diagnostic value of systematic biopsy methods in the investigation of prostate cancer: A systematic review. J Urol 175(5): 1605-1612, 2006. PMID: 16600713. DOI: 10.1016/ S0022-5347(05)00957-2

35 Hotker AM, Mazaheri Y, Zheng JT, Moskowitz CS, Berkowitz J, Lantos JE, Pei X, Zelefsky MJ, Hricak H and Akin O: Prostate cancer: Assessing the effects of androgen-deprivation therapy using quantitative diffusion-weighted and dynamic contrastenhanced mri. Eur Radiol 25(9): 2665-2672, 2015. PMID: 25820537. DOI: $10.1007 / \mathrm{s} 00330-015-3688-1$

36 Song I, Kim CK, Park BK and Park W: Assessment of response to radiotherapy for prostate cancer: Value of diffusion-weighted mri at 3 t. Am J Roentgenol 194(6): W477-W482, 2010. PMID: 20489065. DOI: 10.2214/Ajr.09.3557

37 Lin YC, Lin G, Hong JH, Lin YP, Chen FH, Ng SH and Wang $\mathrm{CC}$ : Diffusion radiomics analysis of intratumoral heterogeneity in a murine prostate cancer model following radiotherapy: Pixelwise correlation with histology. J Magn Reson Imaging 46(2): 483-489, 2017. PMID: 28176411. DOI: 10.1002/jmri. 25583

38 Turchan WT, Kauffmann G, Patel P, Oto A and Liauw SL: Pirads score is associated with biochemical control and distant metastasis in men with intermediate-risk and high-risk prostate cancer treated with radiation therapy. Urol Oncol, 2020. PMID: 31953005. DOI: 10.1016/j.urolonc.2019.12.015

39 Joseph T, McKenna DA, Westphalen AC, Coakley FV, Zhao SJ, Lu Y, Hsu IC, Roach M and Kurhanewicz J: Pretreatment endorectal magnetic resonance imaging and magnetic resonance spectroscopic imaging features of prostate cancer as predictors of response to external beam radiotherapy. Int J Radiat Oncol 
Biol Phys 73(3): 665-671, 2009. PMID: 18760545. DOI: 10.1016/j.ijrobp.2008.04.056

40 Zamboglou C, Drendel V, Jilg CA, Rischke HC, Beck TI, Schultze-Seemann W, Krauss T, Mix M, Schiller F, Wetterauer U, Werner M, Langer M, Bock M, Meyer PT and Grosu AL: Comparison of 68ga-hbed-cc psma-pet/ct and multiparametric mri for gross tumour volume detection in patients with primary prostate cancer based on slice by slice comparison with histopathology. Theranostics 7(1): 228-237, 2017. PMID: 5196899. DOI: $10.7150 /$ thno. 16638
41 Eiber M, Weirich G, Holzapfel K, Souvatzoglou M, Haller B, Rauscher I, Beer AJ, Wester HJ, Gschwend J, Schwaiger M and Maurer T: Simultaneous 68ga-psma hbed-cc pet/mri improves the localization of primary prostate cancer. Eur Urol 70(5): 829-836, 2016. PMID: 26795686. DOI: 10.1016/j.eururo.2015.12.053

Received July 15, 2020

Revised July 29, 2020 Accepted August 5, 2020 